\title{
Mortality Among Persons with Both Asthma and Chronic Obstructive Pulmonary Disease Aged $\geq 25$ Years, by Industry and Occupation - United States, 1999-2016
}

\author{
Katelynn E. Dodd, $\mathrm{MPH}^{1}$; John Wood, $\mathrm{MS}^{1}$; Jacek M. Mazurek, MD, $\mathrm{PhD}^{1}$
}

Patients with asthma typically have chronic airway inflammation, variable airflow limitation, and intermittent respiratory symptoms; patients with chronic obstructive pulmonary disease (COPD) often have fixed airflow limitation and persistent respiratory symptoms. Some patients exhibit features suggesting that they have both conditions, which is termed asthma-COPD overlap. These patients have been reported to have worse health outcomes than do those with asthma or COPD alone (1). To describe mortality among persons aged $\geq 25$ years with asthma-COPD overlap, CDC analyzed 1999-2016 National Vital Statistics multiple-cause-of-death mortality data* extracted from the National Occupational Mortality System (NOMS), which included industry and occupation $^{\dagger}$ information collected from 26 states $^{\S}$ for the years 1999, 2003, 2004, and 2007-2014. Age-adjusted death rates per one million persons and proportionate mortality ratios (PMRs) $^{* *}$ were calculated. During 1999-2016, 6,738 male decedents (age-adjusted rate per million $=4.30$ ) and 12,028 female decedents (5.59) had both asthma and COPD assigned on their death certificate as the underlying or contributing cause of death. The annual age-adjusted death rate per million among decedents with asthma-COPD overlap declined from

\footnotetext{
* https://www.cdc.gov/nchs/data_access/Vitalstatsonline.htm.

$\dagger$ Guidelines for reporting industry and occupation on death certificates instruct recorders to report decedent's "kind of business/industry" and "usual occupation" (i.e., "the type of job the individual was engaged in for most of his or her working life").

$\$$ Colorado, Florida, Georgia, Hawaii, Idaho, Indiana, Kansas, Kentucky, Louisiana, Michigan, Nebraska, Nevada, New Hampshire, New Jersey, New Mexico, North Carolina, North Dakota, Ohio, Rhode Island, South Carolina, Texas, Utah, Vermont, Washington, West Virginia, and Wisconsin. States represent the state where the death took place, not necessarily where the decedent had resided.

Age-adjusted death rates were calculated by applying age-specific death rates to the 2000 U.S. Census standard population age distribution. https://wonder. cdc.gov/wonder/help/mcd.html\#Age-AdjustedRates.

** PMR was defined as the observed number of deaths from asthma-COPD overlap in a specified industry or occupation, divided by the expected number of deaths from asthma-COPD overlap. The expected number of deaths was the total number of deaths in industry or occupation of interest multiplied by a proportion defined as the number of asthma-COPD overlap deaths in all industries or occupations, divided by the total number of deaths in all industries or occupations. The asthma-COPD overlap PMRs were internally adjusted by 5 -year age groups, sex, and race. CIs were calculated assuming Poisson distribution of the data. A PMR $>1.0$ indicates that there were more deaths associated with the condition in a specified occupation or industry than expected; a PMR $<1.0$ indicates that there were fewer deaths associated with the condition in a specified occupation or industry than expected.
}

6.70 in 1999 to 3.01 in $2016(\mathrm{p}<0.05)$ for men and from 7.71 in 1999 to 4.01 in 2016 ( $\mathrm{p}<0.05)$ for women. Among adults aged 25-64 years, asthma-COPD overlap PMRs, by industry, were significantly elevated among nonpaid workers, nonworkers, and persons working at home for both men (1.72) and women (1.40) and among male food, beverage, and tobacco products workers (2.64). By occupation, asthma-COPD overlap PMRs were significantly elevated among both men (1.98) and women (1.79) who were unemployed, had never worked, or were disabled workers and among women bartenders (3.28) and homemakers (1.34). The association between asthmaCOPD overlap mortality and nonworking status among adults aged 25-64 years suggests that asthma-COPD overlap might be associated with substantial morbidity. Increased risk for asthma-COPD overlap mortality among adults in certain industries and occupations suggests targets for public health interventions (e.g., elimination of or removal from exposures, engineering controls, and workplace smoke-free policies) to prevent asthma and COPD in and out of the workplace.

For this report, 1999-2016 National Vital Statistics System's multiple-cause-of-death data extracted from NOMS were analyzed. Decedents with asthma-COPD overlap were identified using the International Classification of Diseases, Tenth Revision codes from death certificates for which both asthma and $\mathrm{COPD}^{\dagger \dagger}$ were listed as the underlying or contributing cause of death. Death rates per million persons aged $\geq 25$ years were assessed by sex and year and were age-adjusted using the 2000 U.S. Census standard population. Time trends in log-transformed age-adjusted mortality rates were assessed in Joinpoint software ${ }^{\mathbb{S}}$ by performing a sequence of permutation tests using Monte Carlo sampling and the Bonferroni correction for multiple testing. Information on industry and

\footnotetext{
†† International Classification of Diseases, Tenth Revision codes for asthma: J45.0 (predominantly allergic asthma), J45.1 (nonallergic asthma), J45.8 (mixed asthma), J45.9 (asthma, unspecified), J46 (status asthmaticus); and COPD: J40 (bronchitis, not specified as acute or chronic), J41.0 (simple chronic bronchitis), J41.1 (mucopurulent chronic bronchitis), J41.8 (mixed simple and mucopurulent chronic bronchitis), J42 (unspecified chronic bronchitis), J43.0 (MacLeod's syndrome), J43.1 (panlobular emphysema), J43.2 (centrilobular emphysema), J43.8 (other emphysema), J43.9 (emphysema, unspecified), J44.0 (chronic obstructive pulmonary disease with acute lower respiratory infection), J44.1 (chronic obstructive pulmonary disease with acute exacerbation, unspecified), J44.8 (other specified chronic obstructive pulmonary disease), J44.9 (chronic obstructive pulmonary disease, unspecified).

$\$ \$$ https://surveillance.cancer.gov/joinpoint/.
} 
occupation, coded by the National Institute for Occupational Safety and Health using the U.S. Census 2000 Industry and Occupation Classification System, was available from 26 states for the years 1999, 2003, 2004, and 2007-2014.99 PMRs, relative to the expected number of decedents with asthma-COPD overlap, and $95 \%$ confidence intervals (CIs) were generated by industry and occupation for men and women and adjusted for 5-year age groups and race. Joinpoint (version 4.7.0.0; National Cancer Institute) and SAS software (version 9.4; SAS Institute) were used to conduct all statistical analyses.

During 1999-2016, among U.S. decedents aged $\geq 25$ years, a total of 4,689,828 had COPD and 164,731 had asthma

\footnotetext{
99 https://www.cdc.gov/niosh/topics/noms/default.html.
}

assigned on their death certificate as the underlying or contributing cause of death. Among these decedents, 18,766 had both asthma and COPD assigned as the underlying or contributing cause of death (6,738 among men and 12,028 among women). The overall death rate among those with asthma-COPD overlap was 5.03 per million persons (4.30 among men and 5.59 among women). The annual age-adjusted death rate per million for men declined from 6.70 in 1999 to 3.01 in 2016 (annual percent change $[\mathrm{APC}]=-4.82 \%$; $\mathrm{p}<0.05)$ and for women declined from 7.71 in 1999 to 4.01 in 2016 ( $\mathrm{APC}=-3.63 \%$; $\mathrm{p}<0.05)$ (Figure).

Among persons aged 25-64 years in 26 states during 1999, 2003, 2004, and 2007-2014, industry and occupation data were available for $784(99.1 \%)$ of 791 decedents with

FIGURE. Number of asthma and chronic obstructive pulmonary disease (COPD) overlap deaths* and age-adjusted asthma-COPD overlap death rates $^{\dagger}$ among decedents aged $\geq 25$ years, by sex — United States, 1999-2016

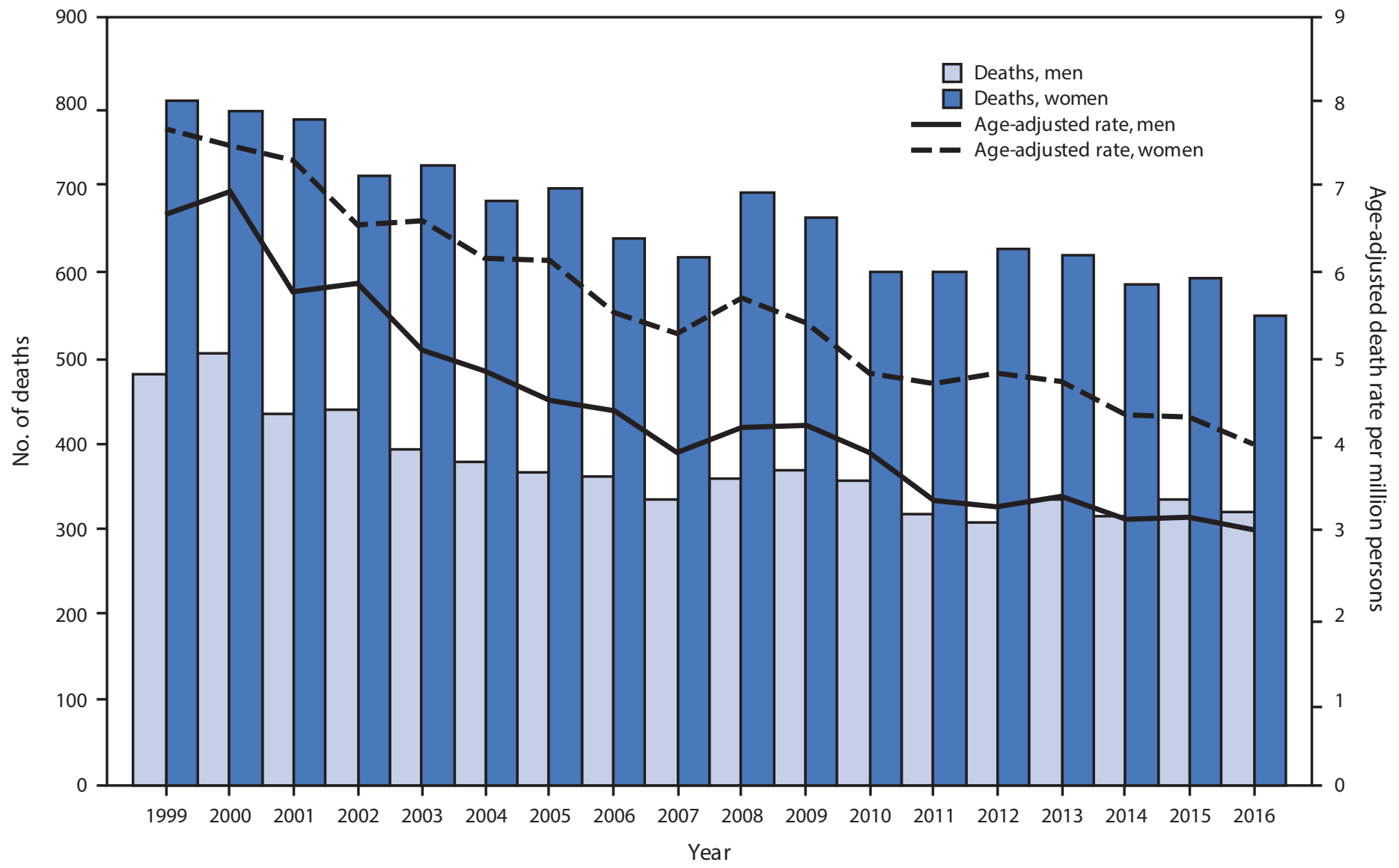

* Decedents with International Classification of Diseases, Tenth Revision codes for asthma: J45.0 (predominantly allergic asthma), J45.1 (nonallergic asthma), J45.8 (mixed asthma), J45.9 (asthma, unspecified), J46 (status asthmaticus); and COPD: J40 (bronchitis, not specified as acute or chronic), J41.0 (simple chronic bronchitis), J41.1 (mucopurulent chronic bronchitis), J41.8 (mixed simple and mucopurulent chronic bronchitis), J42 (unspecified chronic bronchitis), J43.0 (MacLeod's syndrome), J43.1 (panlobular emphysema), J43.2 (centrilobular emphysema), J43.8 (other emphysema), J43.9 (emphysema, unspecified), J44.0 (chronic obstructive pulmonary disease with acute lower respiratory infection), J44.1 (chronic obstructive pulmonary disease with acute exacerbation, unspecified), J44.8 (other specified chronic obstructive pulmonary disease), and $\mathbf{J 4 4 . 9}$ (chronic obstructive pulmonary disease, unspecified) assigned as the underlying cause of death (i.e., the disease or injury which initiated the chain of morbid events leading directly to death, or the circumstances of the accident or violence which produced the fatal injury) or as a contributing cause of death.

${ }^{\dagger}$ Age-adjusted death rates per million persons were calculated by applying age-specific death rates to the 2000 U.S. Census standard population age distribution. https://wonder.cdc.gov/wonder/help/mcd.html\#Age-AdjustedRates. 
asthma-COPD overlap (314 [99.4\%] of 316 men and 470 [98.9\%] of 475 women). By industry, asthma-COPD overlap PMRs were significantly elevated among nonpaid workers, nonworkers, and persons working at home for both men (1.72) and women (1.40) and among male food, beverage, and tobacco products workers (2.64) (Table 1). By occupation, asthma-COPD overlap PMRs were significantly elevated among men (1.98) and women (1.79) who were unemployed, never worked, or were disabled workers and among women bartenders (3.28) and homemakers (1.34) (Table 2).

Among persons aged $\geq 65$ years, industry and occupation data were available for 1,908 (98.3\%) of 1,941 decedents with asthma-COPD overlap (624 [99.5\%] of 627 men and 1,284 [97.7\%] of 1,314 women). Asthma-COPD overlap PMRs were significantly elevated among men in certain industries (e.g., computer and electronic products [2.58]; lumber, wood products, and furniture [2.53]; agriculture, forestry, fishing and hunting [1.82]; beverage manufacturing [3.15]; and miscellaneous manufacturing [1.39)] and among women in private households (1.69), furniture and home furnishings stores (2.99), and unspecified food industries (3.72) (Table 1). By occupation, asthma-COPD overlap PMRs were significantly elevated among men in fishing, hunting, and forestry (3.78); farmers and farm managers (1.62); laborers and material movers (1.54); carpenters (1.68); and industrial production managers (2.23) and among women production workers (1.66) and waitresses (1.70) (Table 2).

\section{Discussion}

Among persons aged $\geq 25$ years, more women than men died from asthma-COPD overlap. A study using 2012 Behavioral Risk Factor Surveillance System data from South Carolina found that asthma-COPD overlap was more prevalent among women than among men (2). The annual age-adjusted death rate per million for both men and women decreased from 1999 through 2016. When analyzed separately, the age-adjusted death rate for asthma similarly declined among men and women from 1999 to 2016 (3). The age-adjusted death rate for COPD among men declined from 1999 to 2011; however, among women, it increased from 2000 to 2011 (4). A 2016 Danish study of the long-term prognosis of persons with chronic airway disease found that the number of deaths from chronic respiratory disease were higher among persons with asthma-COPD overlap with late-onset asthma than among those with COPD only (5).

The American Thoracic Society estimates that approximately $16 \%$ of asthma and $14 \%$ of COPD among adults is attributable to workplace exposures (G). Several workplace exposures, (e.g., dusts, secondhand smoke, welding fumes, and isocyanates) are causative agents for both asthma and $\operatorname{COPD}(7)$. An analysis of workplace exposures among U.S. adults using 2010 National Health Interview Survey data found that workers in industries and occupations similar to those identified in the current study had exposure to vapors, gas, dust, or fumes at work (8). In that study, an estimated $52.9 \%$ of workers in agriculture, forestry, fishing, and hunting and $42.8 \%$ of workers in manufacturing industries, as well as $61.5 \%$ of production workers, $50.8 \%$ of farming, fishing, and forestry workers, and $16.5 \%$ of adults in food preparation and serving occupations had frequent exposure to vapors, gas, dust, or fumes at work (8). Although cigarette smoking is the primary cause of COPD, $25 \%$ of U.S. adults with COPD have never smoked. ${ }^{* * *}$ Among nonsmoking adults in food preparation and serving occupations, an estimated $15.4 \%$ had frequent exposure to secondhand smoke at work (8). Exposure to these agents might explain the increased prevalence of asthma-COPD overlap mortality among workers in certain industries and occupations and should be considered for targets for public health interventions.

Nonpaid workers, nonworkers, and persons working at home had significantly elevated asthma-COPD overlap PMRs among both men and women aged 25-64 years, suggesting that asthma-COPD overlap might be associated with substantial morbidity resulting in loss of employment. Previous reports have similarly found that patients with asthma-COPD overlap were observed to have worse health outcomes than those with asthma or COPD alone $(1,5)$. Moreover, persons with asthma caused or made worse by workplace exposures were similarly more likely to be unemployed and retire at a significantly younger mean age than were those with asthma that is not work-related (9). Retired and unemployed persons might have left the workforce because of severe asthma or COPD; however, complete decedent work histories were unavailable to assess such changes in employment.

The findings in this report are subject to at least five limitations. First, a discrete diagnosis code for asthma-COPD overlap does not currently exist, and no information was available to validate asthma and COPD diagnoses, which might be subject to misdiagnosis. A 1991 study from the United States found that $37 \%$ of subjects with a history of physician-diagnosed airways obstructive disease had airways obstructive disease reported on their death certificate, suggesting the potential for underreporting (10). In addition, it is possible that differences in patterns of asthma and COPD diagnosis regionally and over time might have affected how these diagnoses were recorded on death certificates. Second, discrete diagnosis codes for occupational asthma or COPD do not currently exist; therefore, determining whether the asthma or COPD diagnoses listed as underlying or contributing to death were

\footnotetext{
*** https://www.cdc.gov/tobacco/campaign/tips/diseases/copd.html.
} 
TABLE 1. Industries with five or more asthma-COPD overlap deaths* among decedents aged $\geq 25$ years, by sex and age group -26 states, ${ }^{\dagger}$ 1999, 2003, 2004, and 2007-2014

\begin{tabular}{|c|c|c|c|c|}
\hline \multirow[b]{2}{*}{ Industry } & \multicolumn{2}{|c|}{ Decedents aged $25-64$ yrs } & \multicolumn{2}{|c|}{ Decedents aged $\geq 65$ yrs } \\
\hline & Deaths & $\operatorname{PMR}^{\S}(95 \% \mathrm{Cl})$ & Deaths & $\operatorname{PMR}^{\S}(95 \% \mathrm{Cl})$ \\
\hline \multicolumn{5}{|l|}{ Male } \\
\hline Computer and electronic products" & $\mathrm{N} / \mathrm{A}$ & $\mathrm{N} / \mathrm{A}$ & 10 & $2.58(1.24-4.74)^{* *}$ \\
\hline Lumber, wood products, and furniture & $\mathrm{N} / \mathrm{A}$ & $\mathrm{N} / \mathrm{A}$ & 12 & $2.53(1.30-4.41)^{* *}$ \\
\hline Sawmills and wood preservation ${ }^{\dagger \dagger}$ & $\mathrm{N} / \mathrm{A}$ & $\mathrm{N} / \mathrm{A}$ & 5 & $3.57(1.16-8.34)^{* * *}$ \\
\hline Agriculture, forestry, fishing and hunting? & $\mathrm{N} / \mathrm{A}$ & $\mathrm{N} / \mathrm{A}$ & 66 & $1.82(1.42-2.33)^{* *}$ \\
\hline Logging ${ }^{\dagger \dagger}$ & $\mathrm{N} / \mathrm{A}$ & $\mathrm{N} / \mathrm{A}$ & 11 & $4.82(2.41-8.62)^{* *}$ \\
\hline Animal production ${ }^{\dagger+}$ & $\mathrm{N} / \mathrm{A}$ & $\mathrm{N} / \mathrm{A}$ & 16 & $1.63(0.93-2.65)$ \\
\hline Crop production ${ }^{\dagger \dagger}$ & $\mathrm{N} / \mathrm{A}$ & $\mathrm{N} / \mathrm{A}$ & 35 & $1.59(1.10-2.21)^{* *}$ \\
\hline Broadcasting and telecommunications" & $\mathrm{N} / \mathrm{A}$ & $\mathrm{N} / \mathrm{A}$ & 11 & $1.40(0.70-2.50)$ \\
\hline Wired telecommunications carriers ${ }^{\dagger \dagger}$ & $\mathrm{N} / \mathrm{A}$ & $\mathrm{N} / \mathrm{A}$ & 8 & $1.51(0.65-2.97)$ \\
\hline Personal and laundry services & $\mathrm{N} / \mathrm{A}$ & $\mathrm{N} / \mathrm{A}$ & 7 & $1.37(0.55-2.82)$ \\
\hline Nonmetallic mineral products" & $\mathrm{N} / \mathrm{A}$ & $\mathrm{N} / \mathrm{A}$ & 5 & $1.30(0.42-3.04)$ \\
\hline Wholesale trade & $\mathrm{N} / \mathrm{A}$ & $\mathrm{N} / \mathrm{A}$ & 16 & $1.24(0.71-2.01)$ \\
\hline Groceries and related product wholesalers ${ }^{\dagger \dagger}$ & $\mathrm{N} / \mathrm{A}$ & $\mathrm{N} / \mathrm{A}$ & 5 & $1.99(0.64-4.65)$ \\
\hline Paper and printing & $\mathrm{N} / \mathrm{A}$ & $\mathrm{N} / \mathrm{A}$ & 9 & $1.20(0.55-2.29)$ \\
\hline Printing and related support activities ${ }^{\dagger \dagger}$ & $\mathrm{N} / \mathrm{A}$ & $\mathrm{N} / \mathrm{A}$ & 6 & $1.56(0.57-3.39)$ \\
\hline Publishing, and motion picture and sound recording industries & $\mathrm{N} / \mathrm{A}$ & $\mathrm{N} / \mathrm{A}$ & 5 & $1.16(0.37-2.70)$ \\
\hline Primary metal industries & $\mathrm{N} / \mathrm{A}$ & $\mathrm{N} / \mathrm{A}$ & 14 & $1.09(0.60-1.83)$ \\
\hline Iron and steel mills and steel product manufacturing ${ }^{\dagger \dagger}$ & $\mathrm{N} / \mathrm{A}$ & $\mathrm{N} / \mathrm{A}$ & 10 & $1.07(0.52-1.97)$ \\
\hline Utilities & $\mathrm{N} / \mathrm{A}$ & $\mathrm{N} / \mathrm{A}$ & 11 & $1.03(0.52-1.84)$ \\
\hline Food, beverage, and tobacco products" & 10 & $2.64(1.27-4.86)^{* *}$ & 12 & $1.11(0.57-1.93)$ \\
\hline Beverage manufacturing ${ }^{\dagger \dagger}$ & $\mathrm{N} / \mathrm{A}$ & $\mathrm{N} / \mathrm{A}$ & 5 & $3.15(1.02-7.37)^{* *}$ \\
\hline Arts, entertainment and recreation? & $\mathrm{N} / \mathrm{A}$ & $\mathrm{N} / \mathrm{A}$ & 6 & $0.98(0.36-2.12)$ \\
\hline Retired, unemployed, or nonpaid worker & 38 & $1.71(1.21-2.35)^{* *}$ & 11 & $0.94(0.47-1.68)$ \\
\hline Nonpaid worker or nonworker or own home/at home ${ }^{\dagger \dagger}$ & 38 & $1.72(1.22-2.36)^{* *}$ & 11 & $0.98(0.49-1.75)$ \\
\hline Unknown or not reported & 24 & $1.50(0.96-2.24)$ & 18 & $1.18(0.70-1.86)$ \\
\hline Mining & $\mathrm{N} / \mathrm{A}$ & $N / A$ & 11 & $0.90(0.45-1.61)$ \\
\hline Oil and gas extraction ${ }^{\dagger+}$ & $\mathrm{N} / \mathrm{A}$ & $\mathrm{N} / \mathrm{A}$ & 6 & $1.91(0.70-4.16)$ \\
\hline Finance and Insurance & $\mathrm{N} / \mathrm{A}$ & $\mathrm{N} / \mathrm{A}$ & 13 & $0.86(0.46-1.46)$ \\
\hline Banking and related activities ${ }^{\dagger \dagger}$ & $\mathrm{N} / \mathrm{A}$ & $\mathrm{N} / \mathrm{A}$ & 5 & $1.28(0.42-3.00)$ \\
\hline Insurance carriers and related activities ${ }^{\dagger \dagger}$ & $\mathrm{N} / \mathrm{A}$ & $\mathrm{N} / \mathrm{A}$ & 6 & $0.77(0.28-1.67)$ \\
\hline Home furnishings, appliances, building materials, hardware, lawn and garden? & 5 & $1.49(0.48-3.48)$ & 5 & $0.60(0.19-1.40)$ \\
\hline Motor vehicle and parts dealers & 6 & $1.44(0.53-3.14)$ & 5 & $0.58(0.19-1.36)$ \\
\hline Automobile dealers ${ }^{\dagger \dagger}$ & 5 & $1.94(0.63-4.53)$ & $\mathrm{N} / \mathrm{A}$ & $N / A$ \\
\hline Repair and maintenance & 16 & $1.35(0.77-2.19)$ & 15 & $0.94(0.53-1.55)$ \\
\hline Automotive repair and maintenance ${ }^{\dagger \dagger}$ & 12 & $1.36(0.70-2.37)$ & 9 & $0.80(0.37-1.51)$ \\
\hline Military & 5 & $1.29(0.42-3.02)$ & 10 & $0.68(0.32-1.24)$ \\
\hline Other retail trade & 12 & $1.24(0.64-2.17)$ & 21 & $1.04(0.64-1.59)$ \\
\hline Gasoline stations ${ }^{\dagger \dagger}$ & $\mathrm{N} / \mathrm{A}$ & $\mathrm{N} / \mathrm{A}$ & 5 & $2.50(0.81-5.83)$ \\
\hline Not specified retail trade ${ }^{\dagger \dagger}$ & 6 & $1.32(0.48-2.87)$ & 5 & $0.67(0.22-1.56)$ \\
\hline Chemical & $\mathrm{N} / \mathrm{A}$ & $\mathrm{N} / \mathrm{A}$ & 7 & $0.79(0.32-1.63)$ \\
\hline Industrial and miscellaneous chemicals ${ }^{\dagger \dagger}$ & $\mathrm{N} / \mathrm{A}$ & $\mathrm{N} / \mathrm{A}$ & 5 & $0.85(0.27-1.97)$ \\
\hline Food and beverage stores & $\mathrm{N} / \mathrm{A}$ & $\mathrm{N} / \mathrm{A}$ & 6 & $0.75(0.28-1.64)$ \\
\hline Construction & 58 & $1.16(0.89-1.51)$ & 73 & $1.08(0.85-1.37)$ \\
\hline Public administration & 15 & $1.07(0.60-1.76)$ & 41 & $1.00(0.72-1.36)$ \\
\hline Other general government and support ${ }^{\dagger \dagger}$ & 5 & $0.95(0.31-2.23)$ & 16 & $0.97(0.56-1.58)$ \\
\hline Justice, public order, and safety activities ${ }^{\dagger \dagger}$ & 5 & $0.94(0.30-2.19)$ & 9 & $0.73(0.33-1.38)$ \\
\hline Health care & 10 & $1.05(0.51-1.94)$ & 11 & $0.74(0.37-1.32)$ \\
\hline Hospitals ${ }^{\dagger \dagger}$ & 5 & $1.17(0.38-2.72)$ & 7 & $1.19(0.48-2.46)$ \\
\hline Miscellaneous manufacturing? & 11 & $0.90(0.45-1.61)$ & 41 & $1.39(1.00-1.89)^{* *}$ \\
\hline Not specified manufacturing industries ${ }^{\dagger \dagger}$ & 11 & $0.99(0.50-1.77)$ & 38 & $1.38(0.98-1.90)$ \\
\hline Educational services & 7 & $0.83(0.33-1.71)$ & 22 & $0.85(0.53-1.28)$ \\
\hline Elementary and secondary schools ${ }^{\dagger \dagger}$ & $\mathrm{N} / \mathrm{A}$ & $N / A$ & 17 & $0.91(0.53-1.45)$ \\
\hline Colleges and universities, including junior colleges ${ }^{\dagger \dagger}$ & N/A & $\mathrm{N} / \mathrm{A}$ & 5 & $0.78(0.25-1.81)$ \\
\hline Transportation and warehousing? & 19 & $0.76(0.46-1.18)$ & 53 & $1.01(0.77-1.34)$ \\
\hline Truck transportation ${ }^{\dagger \dagger}$ & 15 & $1.17(0.65-1.92)$ & 22 & $1.14(0.71-1.72)$ \\
\hline Water transportation $^{\dagger \dagger}$ & $\mathrm{N} / \mathrm{A}$ & $N / A$ & 5 & $2.73(0.88-6.38)$ \\
\hline Postal service ${ }^{\dagger \dagger}$ & $\mathrm{N} / \mathrm{A}$ & $\mathrm{N} / \mathrm{A}$ & 8 & $0.92(0.40-1.81)$ \\
\hline Professional, scientific, technical and management services & 6 & $0.62(0.23-1.35)$ & 15 & $0.72(0.40-1.18)$ \\
\hline Machinery & $\mathrm{N} / \mathrm{A}$ & $\mathrm{N} / \mathrm{A}$ & 6 & $0.72(0.26-1.56)$ \\
\hline Accommodation and food services & 8 & $0.62(0.27-1.22)$ & 7 & $0.56(0.23-1.16)$ \\
\hline Restaurants and other food services ${ }^{\dagger \dagger}$ & 7 & $0.69(0.28-1.42)$ & 5 & $0.56(0.18-1.32)$ \\
\hline Administrative and support, and waste management services & 7 & $0.58(0.23-1.20)$ & 10 & $0.87(0.42-1.60)$ \\
\hline
\end{tabular}

See table footnotes on page 675 . 
TABLE 1. (Continued) Industries with five or more asthma-COPD overlap deaths* among decedents aged $\geq 25$ years, by sex and age group 26 states, ${ }^{\dagger}$ 1999, 2003, 2004, and 2007-2014

\begin{tabular}{|c|c|c|c|c|}
\hline \multirow[b]{2}{*}{ Industry } & \multicolumn{2}{|c|}{ Decedents aged $25-64$ yrs } & \multicolumn{2}{|c|}{ Decedents aged $\geq 65$ yrs } \\
\hline & Deaths & $\operatorname{PMR}^{\S}(95 \% \mathrm{Cl})$ & Deaths & $\operatorname{PMR}^{\S}(95 \% \mathrm{CI})$ \\
\hline Transportation equipment ${ }^{\mathfrak{9}}$ & 6 & $0.54(0.20-1.18)$ & 17 & $0.48(0.28-0.76)$ \\
\hline Motor vehicles and motor vehicle equipment manufacturing ${ }^{\dagger \dagger}$ & 5 & $0.62(0.20-1.45)$ & 14 & $0.57(0.31-0.95)$ \\
\hline All other industries & 51 & $\mathrm{~N} / \mathrm{A}$ & 22 & $\mathrm{~N} / \mathrm{A}$ \\
\hline \multicolumn{5}{|l|}{ Female } \\
\hline Retired, unemployed, or nonpaid worker & 192 & $1.40(1.21-1.62)^{* *}$ & 532 & $1.06(0.97-1.15)$ \\
\hline Nonpaid worker or nonworker or own home/at home ${ }^{\dagger \dagger}$ & 192 & $1.40(1.22-1.62)^{* *}$ & 529 & $1.05(0.96-1.15)$ \\
\hline Private households & 8 & $1.34(0.58-2.63)$ & 24 & $1.69(1.08-2.51)^{* *}$ \\
\hline Home furnishings, appliances, building materials, hardware, lawn and garden & $\mathrm{N} / \mathrm{A}$ & $\mathrm{N} / \mathrm{A}$ & 10 & $1.69(0.81-3.11)$ \\
\hline Furniture and home furnishings stores ${ }^{\dagger \dagger}$ & $\mathrm{N} / \mathrm{A}$ & $\mathrm{N} / \mathrm{A}$ & 6 & $2.99(1.10-6.52)^{* *}$ \\
\hline Machinery & $\mathrm{N} / \mathrm{A}$ & $\mathrm{N} / \mathrm{A}$ & 5 & $1.53(0.49-3.57)$ \\
\hline Food, beverage, and tobacco products" & $\mathrm{N} / \mathrm{A}$ & $\mathrm{N} / \mathrm{A}$ & 14 & $1.25(0.68-2.09)$ \\
\hline Not specified food industries ${ }^{\dagger \dagger}$ & $\mathrm{N} / \mathrm{A}$ & $\mathrm{N} / \mathrm{A}$ & 5 & $3.72(1.20-8.68)^{* * *}$ \\
\hline Paper and printing & $\mathrm{N} / \mathrm{A}$ & $\mathrm{N} / \mathrm{A}$ & 7 & $1.21(0.49-2.50)$ \\
\hline Utilities 9 & $\mathrm{~N} / \mathrm{A}$ & $\mathrm{N} / \mathrm{A}$ & 5 & $1.14(0.37-2.66)$ \\
\hline Agriculture, forestry, fishing and hunting & $\mathrm{N} / \mathrm{A}$ & $\mathrm{N} / \mathrm{A}$ & 11 & $1.13(0.57-2.02)$ \\
\hline Crop production ${ }^{\dagger+}$ & $\mathrm{N} / \mathrm{A}$ & $\mathrm{N} / \mathrm{A}$ & 7 & $1.14(0.46-2.36)$ \\
\hline Textile mill, apparel and other finished textile products" & $\mathrm{N} / \mathrm{A}$ & $\mathrm{N} / \mathrm{A}$ & 26 & $1.13(0.74-1.65)$ \\
\hline Cut and sew apparel manufacturing ${ }^{\dagger \dagger}$ & $\mathrm{N} / \mathrm{A}$ & $\mathrm{N} / \mathrm{A}$ & 16 & $1.14(0.65-1.84)$ \\
\hline Fabric mills, except knitting ${ }^{\dagger \dagger}$ & $\mathrm{N} / \mathrm{A}$ & $\mathrm{N} / \mathrm{A}$ & 6 & $0.91(0.33-1.99)$ \\
\hline Electrical equipment, appliances, and components" & $\mathrm{N} / \mathrm{A}$ & $\mathrm{N} / \mathrm{A}$ & 5 & $1.08(0.35-2.51)$ \\
\hline Publishing, and motion picture and sound recording industries & $\mathrm{N} / \mathrm{A}$ & $\mathrm{N} / \mathrm{A}$ & 6 & $1.01(0.37-2.19)$ \\
\hline Administrative and support, and waste management services & 13 & $1.25(0.66-2.13)$ & 12 & $0.72(0.37-1.26)$ \\
\hline Business support services ${ }^{\dagger \dagger}$ & 5 & $1.88(0.61-4.39)$ & 5 & $0.86(0.28-2.01)$ \\
\hline Arts, entertainment and recreation? & 6 & $1.10(0.40-2.40)$ & 6 & $0.65(0.24-1.41)$ \\
\hline Independent artists, performing arts, spectator sports, and related industries ${ }^{\dagger \dagger}$ & 5 & $2.24(0.73-5.24)$ & $\mathrm{N} / \mathrm{A}$ & $\mathrm{N} / \mathrm{A}$ \\
\hline Unknown or not reported & 16 & $1.09(0.63-1.78)$ & 19 & $0.90(0.54-1.41)$ \\
\hline Broadcasting and telecommunications" & 5 & $1.09(0.35-2.54)$ & 13 & $0.92(0.49-1.57)$ \\
\hline Wired telecommunications carriers ${ }^{\dagger \dagger}$ & $\mathrm{N} / \mathrm{A}$ & $\mathrm{N} / \mathrm{A}$ & 10 & $0.92(0.44-1.68)$ \\
\hline Transportation equipment & $\mathrm{N} / \mathrm{A}$ & $\mathrm{N} / \mathrm{A}$ & 15 & $0.87(0.49-1.44)$ \\
\hline Motor vehicles and motor vehicle equipment manufacturing ${ }^{\dagger \dagger}$ & $\mathrm{N} / \mathrm{A}$ & $\mathrm{N} / \mathrm{A}$ & 10 & $0.86(0.42-1.59)$ \\
\hline Chemicaln & $\mathrm{N} / \mathrm{A}$ & $\mathrm{N} / \mathrm{A}$ & 5 & $0.82(0.26-1.91)$ \\
\hline Food and beverage stores & 6 & $1.00(0.37-2.18)$ & 19 & $1.34(0.81-2.10)$ \\
\hline Grocery stores ${ }^{\dagger \dagger}$ & 6 & $1.09(0.40-2.36)$ & 15 & $1.19(0.67-1.97)$ \\
\hline Other retail trade & 22 & $0.97(0.61-1.47)$ & 50 & $0.77(0.57-1.01)$ \\
\hline Clothing and accessories, except shoe, stores ${ }^{\dagger \dagger}$ & $\mathrm{N} / \mathrm{A}$ & N/A & 5 & $0.87(0.28-2.04)$ \\
\hline Department stores ${ }^{\dagger \dagger}$ & $\mathrm{N} / \mathrm{A}$ & N/A & 8 & $0.85(0.37-1.68)$ \\
\hline Not specified retail trade ${ }^{\dagger \dagger}$ & 12 & $0.99(0.51-1.73)$ & 19 & $0.60(0.36-0.94)$ \\
\hline Health careq & 62 & $0.97(0.75-1.26)$ & 114 & $0.99(0.82-1.20)$ \\
\hline Outpatient care centers ${ }^{\dagger \dagger}$ & 11 & $0.91(0.45-1.63)$ & 22 & $1.06(0.66-1.60)$ \\
\hline Other health care services ${ }^{\dagger \dagger}$ & 9 & $1.40(0.64-2.66)$ & 10 & $1.04(0.50-1.92)$ \\
\hline Hospitals ${ }^{\dagger \dagger}$ & 26 & $0.96(0.63-1.41)$ & 59 & $1.00(0.77-1.30)$ \\
\hline Nursing care facilities ${ }^{\dagger \dagger}$ & 9 & $1.15(0.53-2.19)$ & 10 & $0.92(0.44-1.69)$ \\
\hline Miscellaneous manufacturing & 10 & $0.95(0.46-1.75)$ & 41 & $1.25(0.90-1.70)$ \\
\hline Not specified manufacturing industries ${ }^{\dagger \dagger}$ & 9 & $0.98(0.45-1.86)$ & 37 & $1.26(0.89-1.73)$ \\
\hline Real estate and rental leasing? & 5 & $0.93(0.30-2.18)$ & 13 & $0.97(0.52-1.67)$ \\
\hline Real estate ${ }^{\dagger \dagger}$ & 5 & $0.98(0.32-2.28)$ & 13 & $0.99(0.53-1.70)$ \\
\hline Transportation and warehousing & 9 & $0.87(0.40-1.64)$ & 14 & $0.81(0.44-1.36)$ \\
\hline Truck transportation ${ }^{\dagger \dagger}$ & $\mathrm{N} / \mathrm{A}$ & $N / A$ & 6 & $1.91(0.70-4.16)$ \\
\hline Postal service ${ }^{\dagger \dagger}$ & $\mathrm{N} / \mathrm{A}$ & $\mathrm{N} / \mathrm{A}$ & 5 & $1.06(0.34-2.47)$ \\
\hline Social assistance & 7 & $0.83(0.33-1.70)$ & 8 & $0.78(0.33-1.53)$ \\
\hline Accommodation and food services & 22 & $0.82(0.51-1.24)$ & 58 & $1.17(0.90-1.52)$ \\
\hline Restaurants and other food services ${ }^{\dagger \dagger}$ & 15 & $0.72(0.40-1.18)$ & 51 & $1.27(0.96-1.69)$ \\
\hline Traveler accommodation ${ }^{\dagger \dagger}$ & $\mathrm{N} / \mathrm{A}$ & $\mathrm{N} / \mathrm{A}$ & 5 & $0.69(0.22-1.61)$ \\
\hline Personal and laundry services" & 7 & $0.81(0.33-1.67)$ & 23 & $1.12(0.71-1.68)$ \\
\hline Beauty salons ${ }^{\dagger \dagger}$ & 7 & $1.24(0.50-2.55)$ & 14 & $1.04(0.57-1.74)$ \\
\hline Drycleaning and laundry services ${ }^{\dagger \dagger}$ & $\mathrm{N} / \mathrm{A}$ & $\mathrm{N} / \mathrm{A}$ & 7 & $1.47(0.59-3.03)$ \\
\hline Professional, scientific, technical and management services & 12 & $0.80(0.41-1.39)$ & 24 & $0.88(0.56-1.30)$ \\
\hline Legal services ${ }^{\dagger \dagger}$ & $\mathrm{N} / \mathrm{A}$ & $\mathrm{N} / \mathrm{A}$ & 8 & $1.19(0.51-2.33)$ \\
\hline Accounting, tax preparation, bookkeeping and payroll services ${ }^{\dagger \dagger}$ & 5 & $1.31(0.42-3.05)$ & 7 & $0.75(0.30-1.54)$ \\
\hline Public administration? & 13 & $0.74(0.39-1.27)$ & 49 & $1.09(0.81-1.45)$ \\
\hline National security and international affairs ${ }^{\dagger \dagger}$ & $\mathrm{N} / \mathrm{A}$ & $\mathrm{N} / \mathrm{A}$ & 5 & $1.44(0.47-3.36)$ \\
\hline Administration of human resource programs ${ }^{\dagger \dagger}$ & $\mathrm{N} / \mathrm{A}$ & $\mathrm{N} / \mathrm{A}$ & 5 & $1.38(0.45-3.22)$ \\
\hline Justice, public order, and safety activities ${ }^{\dagger \dagger}$ & $\mathrm{N} / \mathrm{A}$ & $\mathrm{N} / \mathrm{A}$ & 6 & $0.87(0.32-1.89)$ \\
\hline Other general government and support ${ }^{\dagger \dagger}$ & 8 & $1.04(0.45-2.05)$ & 26 & $1.09(0.71-1.60)$ \\
\hline
\end{tabular}

See table footnotes on page 675 . 
TABLE 1. (Continued) Industries with five or more asthma-COPD overlap deaths* among decedents aged $\geq 25$ years, by sex and age group 26 states, ${ }^{\dagger}$ 1999, 2003, 2004, and 2007-2014

\begin{tabular}{|c|c|c|c|c|}
\hline \multirow[b]{2}{*}{ Industry } & \multicolumn{2}{|c|}{ Decedents aged $25-64$ yrs } & \multicolumn{2}{|c|}{ Decedents aged $\geq 65$ yrs } \\
\hline & Deaths & $\mathrm{PMR}^{\S}(95 \% \mathrm{Cl})$ & Deaths & $\operatorname{PMR}^{\S}(95 \% \mathrm{Cl})$ \\
\hline Finance and insurance & 9 & $0.62(0.29-1.18)$ & 28 & $0.79(0.53-1.15)$ \\
\hline Insurance carriers and related activities ${ }^{\dagger \dagger}$ & 5 & $0.85(0.28-2.00)$ & 8 & $0.60(0.26-1.19)$ \\
\hline Banking and related activities ${ }^{\dagger \dagger}$ & N/A & N/A & 14 & $0.87(0.47-1.46)$ \\
\hline Educational services & 19 & $0.61(0.37-0.96)$ & 86 & $0.87(0.70-1.08)$ \\
\hline Elementary and secondary schools ${ }^{\dagger \dagger}$ & 18 & $0.68(0.40-1.07)$ & 69 & $0.80(0.62-1.01)$ \\
\hline Colleges and universities, including junior colleges ${ }^{\dagger \dagger}$ & N/A & N/A & 16 & $1.71(0.98-2.78)$ \\
\hline Wholesale trade & N/A & N/A & 5 & $0.75(0.24-1.75)$ \\
\hline All other industries" & 27 & N/A & 37 & N/A \\
\hline
\end{tabular}

Abbreviations: $\mathrm{Cl}=$ confidence interval; $\mathrm{N} / \mathrm{A}=$ not applicable; $\mathrm{PMR}=$ proportionate mortality ratio.

* Decedents with International Classification of Diseases, Tenth Revision codes for asthma: J45.0 (predominantly allergic asthma), J45.1 (nonallergic asthma), J45.8 (mixed asthma), J45.9 (asthma, unspecified), J46 (status asthmaticus); and COPD: J40 (bronchitis, not specified as acute or chronic), J41.0 (simple chronic bronchitis), J41.1 (mucopurulent chronic bronchitis), J41.8 (mixed simple and mucopurulent chronic bronchitis), J42 (unspecified chronic bronchitis), J43.0 (MacLeod's syndrome), J43.1 (panlobular emphysema), J43.2 (centrilobular emphysema), J43.8 (other emphysema), J43.9 (emphysema, unspecified), J44.0 (chronic obstructive pulmonary disease with acute lower respiratory infection), J44.1 (chronic obstructive pulmonary disease with acute exacerbation, unspecified), J44.8 (other specified chronic obstructive pulmonary disease), $\mathbf{J 4 4 . 9}$ (chronic obstructive pulmonary disease, unspecified) assigned as the underlying cause of death (i.e., the disease or injury which initiated the chain of morbid events leading directly to death, or the circumstances of the accident or violence which produced the fatal injury) or as a contributing cause of death.

† Colorado, Florida, Georgia, Hawaii, Idaho, Indiana, Kansas, Kentucky, Louisiana, Michigan, Nebraska, Nevada, New Hampshire, New Jersey, New Mexico, North Carolina, North Dakota, Ohio, Rhode Island, South Carolina, Texas, Utah, Vermont, Washington, West Virginia, and Wisconsin.

$\S$ PMR was defined as the observed number of deaths from asthma-COPD overlap in a specified industry or occupation, divided by the expected number of deaths from asthma-COPD overlap. The expected number of deaths was the total number of deaths in industry or occupation of interest multiplied by a proportion defined as the number of asthma-COPD overlap deaths in all industries or occupations, divided by the total number of deaths in all industries or occupations. The asthmaCOPD overlap PMRs were internally adjusted by 5 -year age groups, sex, and race. Cls were calculated assuming Poisson distribution of the data. A PMR $>1.0$ indicates that there were more deaths associated with the condition in a specified occupation or industry than expected; a PMR $<1.0$ indicates that there were fewer deaths associated with the condition in a specified occupation or industry than expected.

ף U.S. Census 2000 Industry Classification System two-digit industries with five or more deaths.

** Statistically significantly elevated PMR.

t+ U.S. Census 2000 Industry Classification System three-digit industry groups with five or more deaths.

TABLE 2. Occupations with five or more asthma-COPD overlap deaths* among decedents aged $\geq 25$ years, by sex and age group -26 states, ${ }^{\dagger}$ $1999,2003,2004,2007-2014$

\begin{tabular}{|c|c|c|c|c|}
\hline \multirow[b]{2}{*}{ Occupation } & \multicolumn{2}{|c|}{ Decedents aged $25-64$ yrs } & \multicolumn{2}{|c|}{ Decedents aged $\geq 65$ yrs } \\
\hline & Deaths & $\mathrm{PMR}^{\S}(95 \% \mathrm{CI})$ & Deaths & $\mathrm{PMR}^{\S}(95 \% \mathrm{Cl})$ \\
\hline \multicolumn{5}{|l|}{ Male } \\
\hline Fishing, hunting, and forestry occupations" & $\mathrm{N} / \mathrm{A}$ & $\mathrm{N} / \mathrm{A}$ & 10 & $3.78(1.82-6.95)^{* *}$ \\
\hline Logging workers ${ }^{\dagger \dagger}$ & N/A & $\mathrm{N} / \mathrm{A}$ & 10 & $5.64(2.71-10.37)^{* *}$ \\
\hline Farmers and farm managers? & $\mathrm{N} / \mathrm{A}$ & $\mathrm{N} / \mathrm{A}$ & 43 & $1.62(1.17-2.18)^{* *}$ \\
\hline Farmers and ranchers ${ }^{\dagger \dagger}$ & $\mathrm{N} / \mathrm{A}$ & $\mathrm{N} / \mathrm{A}$ & 43 & $1.67(1.21-2.25)^{* *}$ \\
\hline Food processing workers & N/A & $\mathrm{N} / \mathrm{A}$ & 6 & $1.59(0.58-3.47)$ \\
\hline Textile, apparel, and furnishings workers & $\mathrm{N} / \mathrm{A}$ & $\mathrm{N} / \mathrm{A}$ & 6 & $1.56(0.57-3.39)$ \\
\hline Retired, students, volunteers, homemakers and unemployed? & 40 & $1.77(1.26-2.41)^{* *}$ & 11 & $0.89(0.44-1.59)$ \\
\hline Unemployed, never worked, disabled ${ }^{\dagger \dagger}$ & 36 & $1.98(1.39-2.75)^{* *}$ & 8 & $1.38(0.59-2.71)$ \\
\hline Vehicle and mobile equipment mechanics, installers, and repairers & 15 & $1.41(0.79-2.33)$ & 17 & $1.06(0.62-1.69)$ \\
\hline Automotive service technicians and mechanics ${ }^{\dagger \dagger}$ & 7 & $1.03(0.41-2.13)$ & 6 & $0.71(0.26-1.54)$ \\
\hline Unknown or not reported & 18 & $1.30(0.77-2.05)$ & 15 & $0.97(0.54-1.60)$ \\
\hline Metal workers and plastic workers? & 13 & $1.27(0.67-2.17)$ & 25 & $0.93(0.60-1.37)$ \\
\hline Welding, soldering, and brazing workers ${ }^{\dagger \dagger}$ & 5 & $1.31(0.42-3.06)$ & $\mathrm{N} / \mathrm{A}$ & $\mathrm{N} / \mathrm{A}$ \\
\hline Tool and die makers ${ }^{\dagger \dagger}$ & $\mathrm{N} / \mathrm{A}$ & $N / A$ & 5 & $1.32(0.43-3.09)$ \\
\hline Metalworkers and plastic workers, all other ${ }^{\dagger \dagger}$ & $\mathrm{N} / \mathrm{A}$ & $\mathrm{N} / \mathrm{A}$ & 6 & $2.45(0.90-5.34)$ \\
\hline Machinists ${ }^{\dagger \dagger}$ & N/A & $\mathrm{N} / \mathrm{A}$ & 10 & $0.99(0.48-1.83)$ \\
\hline Laborers and material movers, hand & 21 & $1.26(0.78-1.92)$ & 35 & $1.54(1.07-2.14)^{* *}$ \\
\hline Laborers and freight, stock, and material movers, hand ${ }^{\dagger \dagger}$ & 21 & $1.34(0.82-2.04)$ & 32 & $1.47(1.01-2.08)^{* *}$ \\
\hline Agricultural workers, including supervisors? & N/A & N/A & 7 & $1.53(0.62-3.16)$ \\
\hline Miscellaneous agricultural workers ${ }^{\dagger \dagger}$ & $\mathrm{N} / \mathrm{A}$ & $\mathrm{N} / \mathrm{A}$ & 6 & $1.58(0.58-3.43)$ \\
\hline Rail and water transportation workers & $\mathrm{N} / \mathrm{A}$ & $\mathrm{N} / \mathrm{A}$ & 7 & $1.48(0.59-3.04)$ \\
\hline Motor vehicle operators & 23 & $1.19(0.75-1.78)$ & 38 & $1.22(0.86-1.67)$ \\
\hline Bus drivers ${ }^{\dagger \dagger}$ & N/A & $\mathrm{N} / \mathrm{A}$ & 5 & $1.77(0.57-4.12)$ \\
\hline Driver-sales workers and truck drivers ${ }^{\dagger \dagger}$ & 21 & $1.24(0.76-1.89)$ & 32 & $1.19(0.81-1.68)$ \\
\hline Other material moving workers, except laborers & $\mathrm{N} / \mathrm{A}$ & $\mathrm{N} / \mathrm{A}$ & 7 & $1.21(0.49-2.50)$ \\
\hline Other production occupations, including supervisors? & 13 & $1.17(0.62-2.00)$ & 23 & $0.73(0.46-1.10)$ \\
\hline First-line supervisors or managers of production and operating workers ${ }^{\dagger \dagger}$ & N/A & N/A & 9 & $0.83(0.38-1.58)$ \\
\hline Production workers, all other ${ }^{\dagger \dagger}$ & 6 & $1.27(0.47-2.77)$ & 7 & $0.70(0.28-1.44)$ \\
\hline
\end{tabular}

See table footnotes on page 677. 
TABLE 2. (Continued) Occupations with five or more asthma-COPD overlap deaths* among decedents aged $\geq 25$ years, by sex and age group 26 states, ${ }^{\dagger}$ 1999, 2003, 2004, 2007-2014

\begin{tabular}{|c|c|c|c|c|}
\hline \multirow[b]{2}{*}{ Occupation } & \multicolumn{2}{|c|}{ Decedents aged $25-64$ yrs } & \multicolumn{2}{|c|}{ Decedents aged $\geq 65$ yrs } \\
\hline & Deaths & $\operatorname{PMR}^{\S}(95 \% \mathrm{Cl})$ & Deaths & $\mathrm{PMR}^{\S}(95 \% \mathrm{Cl})$ \\
\hline Construction trades workers" & 45 & $1.11(0.81-1.48)$ & 66 & $1.20(0.94-1.54)$ \\
\hline Carpenters $^{\dagger \dagger}$ & 10 & $1.23(0.59-2.27)$ & 20 & $1.68(1.02-2.59)^{* *}$ \\
\hline Operating engineers and other construction equipment operators ${ }^{\dagger \dagger}$ & $\mathrm{N} / \mathrm{A}$ & $\mathrm{N} / \mathrm{A}$ & 8 & $1.59(0.69-3.13)$ \\
\hline Construction laborers $^{\dagger \dagger}$ & 17 & $1.29(0.75-2.07)$ & 16 & $1.49(0.85-2.41)$ \\
\hline Electricians $^{\dagger \dagger}$ & 7 & $1.93(0.78-3.98)$ & N/A & N/A \\
\hline Financial specialists & $\mathrm{N} / \mathrm{A}$ & $\mathrm{N} / \mathrm{A}$ & 12 & $1.18(0.61-2.06)$ \\
\hline Accountants and auditors ${ }^{\dagger \dagger}$ & $\mathrm{N} / \mathrm{A}$ & N/A & 9 & $1.23(0.57-2.34)$ \\
\hline Business operations specialists" & N/A & $\mathrm{N} / \mathrm{A}$ & 8 & $1.14(0.49-2.24)$ \\
\hline Drafters, engineering, and mapping technicians ${ }^{q}$ & $\mathrm{~N} / \mathrm{A}$ & $\mathrm{N} / \mathrm{A}$ & 5 & $1.07(0.35-2.51)$ \\
\hline Other protective service workers, including supervisors" & $\mathrm{N} / \mathrm{A}$ & $\mathrm{N} / \mathrm{A}$ & 5 & $1.03(0.33-2.40)$ \\
\hline Assemblers and fabricators" & $\mathrm{N} / \mathrm{A}$ & $\mathrm{N} / \mathrm{A}$ & 8 & $1.03(0.44-2.02)$ \\
\hline Miscellaneous assemblers and fabricators ${ }^{\dagger \dagger}$ & $\mathrm{N} / \mathrm{A}$ & N/A & 5 & $1.03(0.33-2.40)$ \\
\hline Law enforcement workers, including supervisors" & $\mathrm{N} / \mathrm{A}$ & N/A & 8 & $1.02(0.44-2.01)$ \\
\hline Police and sheriff's patrol officers ${ }^{\dagger \dagger}$ & $\mathrm{N} / \mathrm{A}$ & N/A & 5 & $1.00(0.33-2.35)$ \\
\hline Extraction workers & N/A & $\mathrm{N} / \mathrm{A}$ & 6 & $1.01(0.37-2.19)$ \\
\hline Health diagnosing and treating practitioners and technical occupations & $\mathrm{N} / \mathrm{A}$ & $\mathrm{N} / \mathrm{A}$ & 5 & $1.00(0.32-2.34)$ \\
\hline Engineers & $\mathrm{N} / \mathrm{A}$ & $\mathrm{N} / \mathrm{A}$ & 22 & $0.95(0.59-1.44)$ \\
\hline Civil engineers ${ }^{\dagger \dagger}$ & $\mathrm{N} / \mathrm{A}$ & $\mathrm{N} / \mathrm{A}$ & 6 & $1.43(0.52-3.11)$ \\
\hline Office and administrative support occupations" & 12 & $1.04(0.54-1.82)$ & 23 & $0.87(0.55-1.30)$ \\
\hline Building and grounds cleaning and maintenance occupations & 15 & $1.03(0.58-1.70)$ & 18 & $0.92(0.55-1.46)$ \\
\hline Janitors and building cleaners ${ }^{\dagger \dagger}$ & 12 & $1.38(0.71-2.40)$ & 12 & $0.85(0.44-1.48)$ \\
\hline Education, training, and library occupations ${ }^{\natural}$ & $\mathrm{N} / \mathrm{A}$ & N/A & 13 & $0.86(0.46-1.48)$ \\
\hline Postsecondary teachers ${ }^{\dagger \dagger}$ & $\mathrm{N} / \mathrm{A}$ & N/A & 5 & $1.20(0.39-2.81)$ \\
\hline Elementary and middle school teachers ${ }^{\dagger \dagger}$ & N/A & $\mathrm{N} / \mathrm{A}$ & 8 & $1.01(0.43-1.98)$ \\
\hline Supervisors, construction and extraction workers & N/A & $\mathrm{N} / \mathrm{A}$ & 6 & $0.78(0.29-1.70)$ \\
\hline $\begin{array}{l}\text { First-line supervisors or managers of construction trades and } \\
\text { extraction workers }{ }^{\dagger \dagger}\end{array}$ & N/A & N/A & 6 & $0.78(0.29-1.70)$ \\
\hline $\begin{array}{l}\text { Electrical equipment mechanics and other installation, maintenance, and } \\
\text { repair workers }\end{array}$ & 9 & $1.01(0.46-1.91)$ & 16 & $0.75(0.43-1.22)$ \\
\hline First-line supervisors or managers of mechanics, installers, and repairers ${ }^{\dagger \dagger}$ & $\mathrm{N} / \mathrm{A}$ & $\mathrm{N} / \mathrm{A}$ & 7 & $1.63(0.65-3.35)$ \\
\hline Food preparation and serving related occupations" & 7 & $0.77(0.31-1.60)$ & N/A & N/A \\
\hline Sales and related occupations & 14 & $0.70(0.38-1.17)$ & 48 & $0.88(0.65-1.17)$ \\
\hline First-line supervisors or managers of nonretail sales workers ${ }^{\dagger \dagger}$ & $\mathrm{N} / \mathrm{A}$ & $\mathrm{N} / \mathrm{A}$ & 6 & $1.20(0.44-2.61)$ \\
\hline Retail salespersons ${ }^{\dagger \dagger}$ & 5 & $0.85(0.27-1.98)$ & 9 & $0.77(0.35-1.46)$ \\
\hline Sales representatives, wholesale and manufacturing ${ }^{\dagger \dagger}$ & $\mathrm{N} / \mathrm{A}$ & $\mathrm{N} / \mathrm{A}$ & 5 & $0.77(0.25-1.80)$ \\
\hline First-line supervisors or managers of retail sales workers ${ }^{\dagger \dagger}$ & N/A & N/A & 13 & $0.72(0.38-1.23)$ \\
\hline Management occupations, except agriculturalף & 11 & $0.53(0.26-0.94)$ & 54 & $0.91(0.69-1.19)$ \\
\hline Managers, all other ${ }^{\dagger \dagger}$ & 6 & $1.19(0.44-2.60)$ & 17 & $1.10(0.64-1.77)$ \\
\hline Industrial production managers ${ }^{\dagger \dagger}$ & $\mathrm{N} / \mathrm{A}$ & N/A & 10 & $2.23(1.07-4.10)^{* *}$ \\
\hline Transportation, storage, and distribution managers ${ }^{\dagger \dagger}$ & $\mathrm{N} / \mathrm{A}$ & N/A & 5 & $1.87(0.60-4.36)$ \\
\hline Chief executives $^{\dagger \dagger}$ & $\mathrm{N} / \mathrm{A}$ & $\mathrm{N} / \mathrm{A}$ & 8 & $1.55(0.67-3.05)$ \\
\hline Military occupations" & $\mathrm{N} / \mathrm{A}$ & N/A & 9 & $0.66(0.30-1.26)$ \\
\hline Military, rank not specified ${ }^{\dagger \dagger}$ & $\mathrm{N} / \mathrm{A}$ & $\mathrm{N} / \mathrm{A}$ & 6 & $1.06(0.39-2.30)$ \\
\hline All other occupations" & 58 & N/A & 42 & N/A \\
\hline \multicolumn{5}{|l|}{ Female } \\
\hline Agricultural workers, including supervisors" & $\mathrm{N} / \mathrm{A}$ & N/A & 5 & $2.00(0.65-4.68)$ \\
\hline Media and communications workers & $\mathrm{N} / \mathrm{A}$ & N/A & 5 & $1.41(0.46-3.29)$ \\
\hline Other protective service workers, including supervisors" & 5 & $2.34(0.76-5.47)$ & N/A & N/A \\
\hline Other production occupations, including supervisors" & N/A & N/A & 39 & $1.29(0.92-1.77)$ \\
\hline Inspectors, testers, sorters, samplers, and weighers ${ }^{\dagger \dagger}$ & $\mathrm{N} / \mathrm{A}$ & $\mathrm{N} / \mathrm{A}$ & 8 & $1.19(0.51-2.35)$ \\
\hline Production workers, all other ${ }^{\dagger+}$ & $\mathrm{N} / \mathrm{A}$ & $\mathrm{N} / \mathrm{A}$ & 22 & $1.66(1.04-2.52)^{* *}$ \\
\hline Textile, apparel, and furnishings workers" & $\mathrm{N} / \mathrm{A}$ & $\mathrm{N} / \mathrm{A}$ & 34 & $1.20(0.83-1.68)$ \\
\hline Sewing machine operators ${ }^{t \dagger}$ & $\mathrm{N} / \mathrm{A}$ & $\mathrm{N} / \mathrm{A}$ & 21 & $1.32(0.82-2.02)$ \\
\hline Tailors, dressmakers, and sewers ${ }^{\dagger \dagger}$ & $\mathrm{N} / \mathrm{A}$ & N/A & 5 & $1.15(0.37-2.68)$ \\
\hline Retired, students, volunteers, homemakers and unemployed? & 193 & $1.41(1.22-1.63)^{* *}$ & 535 & $1.06(0.97-1.15)$ \\
\hline Unemployed, never worked, disabled ${ }^{\dagger \dagger}$ & 41 & $1.79(1.29-2.42)^{* *}$ & 11 & $1.27(0.64-2.27)$ \\
\hline Homemakers $^{\dagger \dagger}$ & 151 & $1.34(1.14-1.58)^{* *}$ & 521 & $1.05(0.97-1.15)$ \\
\hline Farmers and farm managers & N/A & N/A & 5 & $1.02(0.33-2.38)$ \\
\hline Farmers and ranchers ${ }^{\dagger \dagger}$ & $\mathrm{N} / \mathrm{A}$ & N/A & 5 & $1.07(0.35-2.49)$ \\
\hline Financial specialists" & $\mathrm{N} / \mathrm{A}$ & N/A & 14 & $1.00(0.54-1.67)$ \\
\hline Accountants and auditors ${ }^{\dagger \dagger}$ & $\mathrm{N} / \mathrm{A}$ & N/A & 8 & $0.87(0.38-1.72)$ \\
\hline Health technologists and technicians" & 11 & $1.33(0.66-2.38)$ & 9 & $0.66(0.30-1.26)$ \\
\hline Licensed practical and licensed vocational nurses ${ }^{\dagger \dagger}$ & 8 & $2.24(0.96-4.40)$ & $\mathrm{N} / \mathrm{A}$ & $\mathrm{N} / \mathrm{A}$ \\
\hline
\end{tabular}

See table footnotes on page 677. 
TABLE 2. (Continued) Occupations with five or more asthma-COPD overlap deaths* among decedents aged $\geq 25$ years, by sex and age group 26 states, ${ }^{\dagger} 1999,2003,2004,2007-2014$

\begin{tabular}{|c|c|c|c|c|}
\hline \multirow[b]{2}{*}{ Occupation } & \multicolumn{2}{|c|}{ Decedents aged $25-64$ yrs } & \multicolumn{2}{|c|}{ Decedents aged $\geq 65$ yrs } \\
\hline & Deaths & $\mathrm{PMR}^{\S}(95 \% \mathrm{Cl})$ & Deaths & $\mathrm{PMR}^{\S}(95 \% \mathrm{Cl})$ \\
\hline Healthcare support occupations & 27 & $1.27(0.83-1.84)$ & 38 & $1.38(0.98-1.89)$ \\
\hline Nursing, psychiatric, and home health aides ${ }^{\dagger \dagger}$ & 25 & $1.52(0.98-2.25)$ & 31 & $1.42(0.97-2.02)$ \\
\hline Motor vehicle operators" & 6 & $1.25(0.46-2.73)$ & $\mathrm{N} / \mathrm{A}$ & N/A \\
\hline Sales and related occupations" & 31 & $1.00(0.68-1.42)$ & 66 & $0.83(0.65-1.06)$ \\
\hline Cashiers $^{\dagger \dagger}$ & 9 & $1.61(0.74-3.06)$ & $\mathrm{N} / \mathrm{A}$ & N/A \\
\hline Retail salespersons ${ }^{\dagger \dagger}$ & 11 & $1.12(0.56-2.00)$ & 35 & $1.08(0.75-1.50)$ \\
\hline First-line supervisors or managers of retail sales workers ${ }^{\dagger \dagger}$ & $\mathrm{N} / \mathrm{A}$ & N/A & 17 & $0.85(0.49-1.35)$ \\
\hline Building and grounds cleaning and maintenance occupations & 14 & $0.96(0.53-1.62)$ & 34 & $1.13(0.79-1.58)$ \\
\hline Janitors and building cleaners ${ }^{\dagger \dagger}$ & 6 & $1.41(0.52-3.07)$ & 9 & $1.16(0.53-2.20)$ \\
\hline Maids and housekeeping cleaners ${ }^{\dagger \dagger}$ & 8 & $0.90(0.39-1.76)$ & 25 & $1.23(0.79-1.81)$ \\
\hline Personal care and service occupations? & 16 & $0.94(0.54-1.53)$ & 24 & $0.87(0.56-1.29)$ \\
\hline Personal and home care aides ${ }^{\dagger \dagger}$ & 5 & $1.27(0.41-2.97)$ & 5 & $1.07(0.34-2.49)$ \\
\hline Hairdressers, hairstylists, and cosmetologists ${ }^{\dagger \dagger}$ & 6 & $1.16(0.42-2.52)$ & 13 & $1.06(0.56-1.81)$ \\
\hline Community and social services occupations" & 6 & $0.92(0.34-2.01)$ & $\mathrm{N} / \mathrm{A}$ & $\mathrm{N} / \mathrm{A}$ \\
\hline Laborers and material movers, hand" & 8 & $0.92(0.40-1.82)$ & 25 & $1.31(0.85-1.94)$ \\
\hline Laborers and freight, stock, and material movers, hand ${ }^{\dagger \dagger}$ & 8 & $1.01(0.43-1.98)$ & 25 & $1.51(0.97-2.22)$ \\
\hline Unknown or not reported? & 13 & $0.92(0.49-1.57)$ & 23 & $1.15(0.73-1.73)$ \\
\hline Food preparation and serving related occupations" & 18 & $0.83(0.49-1.31)$ & 57 & $1.22(0.93-1.60)$ \\
\hline Cooks $^{\dagger+}$ & 6 & $0.91(0.33-1.98)$ & 23 & $1.26(0.80-1.89)$ \\
\hline Bartenders ${ }^{\dagger \dagger}$ & 6 & $3.28(1.20-7.15)^{* *}$ & $\mathrm{~N} / \mathrm{A}$ & N/A \\
\hline Waiters and waitresses ${ }^{\dagger \dagger}$ & N/A & N/A & 23 & $1.70(1.07-2.55)^{* *}$ \\
\hline Education, training, and library occupations ${ }^{\pi}$ & 17 & $0.82(0.48-1.31)$ & 58 & $0.87(0.67-1.14)$ \\
\hline Elementary and middle school teachers ${ }^{\dagger \dagger}$ & 10 & $0.82(0.39-1.50)$ & 40 & $0.86(0.62-1.18)$ \\
\hline Health diagnosing and treating practitioners and technical occupations ${ }^{\|}$ & 12 & $0.72(0.37-1.25)$ & 43 & $1.09(0.79-1.46)$ \\
\hline Registered nurses ${ }^{\dagger \dagger}$ & 11 & $0.75(0.38-1.34)$ & 41 & $1.11(0.80-1.50)$ \\
\hline Office and administrative support occupations" & 36 & $0.64(0.45-0.88)$ & 152 & $0.86(0.73-1.01)$ \\
\hline First-line supervisors or managers of office and administrative support workers ${ }^{\dagger \dagger}$ & 5 & $0.96(0.31-2.24)$ & 10 & $0.85(0.41-1.57)$ \\
\hline Secretaries and administrative assistants ${ }^{\dagger \dagger}$ & 10 & $0.68(0.33-1.26)$ & 54 & $0.82(0.62-1.08)$ \\
\hline Office clerks, general ${ }^{t \dagger}$ & 7 & $1.04(0.42-2.15)$ & 18 & $0.85(0.50-1.35)$ \\
\hline Receptionists and information clerks ${ }^{\dagger \dagger}$ & $\mathrm{N} / \mathrm{A}$ & N/A & 6 & $1.24(0.45-2.69)$ \\
\hline Telephone operators ${ }^{\dagger \dagger}$ & $\mathrm{N} / \mathrm{A}$ & $\mathrm{N} / \mathrm{A}$ & 7 & $1.18(0.47-2.43)$ \\
\hline Bookkeeping, accounting, and auditing clerks ${ }^{\dagger \dagger}$ & $\mathrm{N} / \mathrm{A}$ & N/A & 24 & $0.89(0.57-1.32)$ \\
\hline Office and administrative support workers, all other ${ }^{\dagger \dagger}$ & $\mathrm{N} / \mathrm{A}$ & $\mathrm{N} / \mathrm{A}$ & 6 & $1.80(0.66-3.92)$ \\
\hline Management occupations, except agricultural" & 11 & $0.45(0.23-0.81)$ & 54 & $1.06(0.80-1.39)$ \\
\hline Property, real estate, and community association managers ${ }^{\dagger \dagger}$ & N/A & $\mathrm{N} / \mathrm{A}$ & 6 & $2.02(0.74-4.40)$ \\
\hline Food service managers ${ }^{\dagger \dagger}$ & N/A & N/A & 10 & $1.14(0.55-2.10)$ \\
\hline Financial managers ${ }^{\dagger \dagger}$ & $\mathrm{N} / \mathrm{A}$ & $\mathrm{N} / \mathrm{A}$ & 5 & $0.95(0.31-2.22)$ \\
\hline Managers, all other ${ }^{\dagger \dagger}$ & $\mathrm{N} / \mathrm{A}$ & $\mathrm{N} / \mathrm{A}$ & 9 & $0.74(0.34-1.41)$ \\
\hline Business operations specialists" & $\mathrm{N} / \mathrm{A}$ & $\mathrm{N} / \mathrm{A}$ & 8 & $0.74(0.32-1.45)$ \\
\hline Assemblers and fabricators & $\mathrm{N} / \mathrm{A}$ & $\mathrm{N} / \mathrm{A}$ & 5 & $0.36(0.12-0.84)$ \\
\hline All other occupations" & 46 & $\mathrm{~N} / \mathrm{A}$ & 51 & N/A \\
\hline
\end{tabular}

Abbreviations: $\mathrm{Cl}=$ confidence interval; $\mathrm{N} / \mathrm{A}=$ not applicable; $\mathrm{PMR}=$ proportionate mortality ratio.

* Decedents with International Classification of Diseases, Tenth Revision codes for asthma: J45.0 (predominantly allergic asthma), J45.1 (nonallergic asthma), J45.8 (mixed asthma), J45.9 (asthma, unspecified), J46 (status asthmaticus); and COPD: J40 (bronchitis, not specified as acute or chronic), J41.0 (simple chronic bronchitis), J41.1 (mucopurulent chronic bronchitis), J41.8 (mixed simple and mucopurulent chronic bronchitis), J42 (unspecified chronic bronchitis), J43.0 (MacLeod's syndrome), J43.1 (panlobular emphysema), J43.2 (centrilobular emphysema), J43.8 (other emphysema), J43.9 (emphysema, unspecified), J44.0 (chronic obstructive pulmonary disease with acute lower respiratory infection), J44.1 (chronic obstructive pulmonary disease with acute exacerbation, unspecified), J44.8 (other specified chronic obstructive pulmonary disease), J44.9 (chronic obstructive pulmonary disease, unspecified) assigned as the underlying cause of death (i.e., the disease or injury which initiated the chain of morbid events leading directly to death, or the circumstances of the accident or violence which produced the fatal injury) or as a contributing cause of death.

† Colorado, Florida, Georgia, Hawaii, Idaho, Indiana, Kansas, Kentucky, Louisiana, Michigan, Nebraska, Nevada, New Hampshire, New Jersey, New Mexico, North Carolina, North Dakota, Ohio, Rhode Island, South Carolina, Texas, Utah, Vermont, Washington, West Virginia, and Wisconsin.

$\S$ PMR was defined as the observed number of deaths from asthma-COPD overlap in a specified industry or occupation, divided by the expected number of deaths from asthma-COPD overlap. The expected number of deaths was the total number of deaths in industry or occupation of interest multiplied by a proportion defined as the number of asthma-COPD overlap deaths in all industries or occupations, divided by the total number of deaths in all industries or occupations. The asthmaCOPD overlap PMRs were internally adjusted by 5 -year age groups, sex, and race. Cls were calculated assuming Poisson distribution of the data. A PMR $>1.0$ indicates that there were more deaths associated with the condition in a specified occupation or industry than expected; a PMR $<1.0$ indicates that there were fewer deaths associated with the condition in a specified occupation or industry than expected.

" U.S. Census 2000 Occupation Classification System two-digit occupations with five or more deaths.

** Statistically significantly elevated PMR.

t† U.S. Census 2000 Occupation Classification System three-digit occupation groups with five or more deaths. 


\section{Summary}

What is already known about this topic?

Patients with features of both asthma and chronic obstructive pulmonary disease (COPD), termed asthma-COPD overlap, have been reported to have worse health outcomes than those with asthma or COPD alone.

What is added by this report?

During 1999-2016, 18,766 U.S. decedents aged $\geq 25$ years had asthma and COPD assigned on their death certificates as the underlying or contributing cause of death. Among adults aged 25-64 years, asthma-COPD overlap mortality was associated with nonworking status among men and women and bartending among women.

What are the implications for public health practice?

Excess risk for asthma-COPD overlap mortality among adults in certain industries and occupations suggests targets for public health interventions to prevent asthma and COPD in and out of the workplace.

caused by workplace exposures is not possible. Third, guidelines for reporting industry and occupation on death certificates ${ }^{\dagger \dagger \dagger}$ instruct recorders to report decedent's "kind of business/ industry" and "usual occupation" (i.e., "the type of job the individual was engaged in for most of his or her working life"). Therefore, if asthma and COPD were caused by workplace exposures, the industry and occupation reported on death certificates might not reflect those in which potential workplace exposures occurred. Workers might have changed jobs or held more than one job; however, information is not available to assess changes in employment. Fourth, no information was available to evaluate the smoking status of decedents, which might have caused or worsened the consequences of asthma or COPD. Finally, only selected states provided information on industry and occupation, and only for certain years; therefore, information by industry and occupation might not be nationally representative.

Among persons aged $\geq 25$ years, deaths associated with asthma-COPD overlap were more frequent among women than among men. The association between asthma-COPD overlap mortality and nonworking status among adults of working age (25-64 years) suggests that asthma-COPD overlap might be associated with substantial morbidity resulting in loss of employment. Increased risk for asthma-COPD overlap mortality among adults in certain industries and occupations suggests targets for public health interventions (e.g., elimination or substitution of exposures, removing workers from exposures, engineering controls such as ventilation or enclosure of exposure generating processes, and workplace smoke-free policies) to prevent asthma and COPD in and out of the workplace. Continued surveillance for asthma-COPD overlap morbidity and mortality is essential to inform policy and intervention activities.

\section{Acknowledgments}

Colorado Department of Public Health and Environment; Florida Department of Health; Georgia Department of Public Health; Hawaii State Department of Health; Idaho Department of Health and Welfare; Indiana State Department of Health; Kansas Department of Health and Environment; Kentucky Department for Public Health; Louisiana Department of Health; Michigan Department of Health \& Human Services; Nebraska Department of Health and Human Services; Nevada Department of Health and Human Services; New Hampshire Department of Health and Human Services; New Jersey Department of Health; New Mexico Department of Health; North Carolina Department of Health and Human Services; North Dakota Department of Health; Ohio Department of Health; Rhode Island Department of Health; South Carolina Department of Health and Environmental Control; Texas Department of State Health Services; Utah Department of Health; Vermont Department of Health; Washington State Department of Health; West Virginia Department of Health and Human Resources; Wisconsin Department of Health Services; Janet Croft, $\mathrm{PhD}$, National Center for Chronic Disease Prevention and Health Promotion, CDC; David Weissman, MD, National Institute for Occupational Safety and Health, CDC.

Corresponding author: Katelynn Dodd, yla8@cdc.gov, 304-285-6305.

\footnotetext{
${ }^{1}$ Respiratory Health Division, National Institute for Occupational Safety and Health, CDC.
}

All authors have completed and submitted the International Committee of Medical Journal Editors form for disclosure of potential conflicts of interest. No potential conflicts of interest were disclosed.

\section{References}

1. Global Initiative for Asthma. Global strategy for asthma management and prevention. Fontana, WI: Global Initiative for Asthma; 2020. https:// ginasthma.org/wp-content/uploads/2020/04/GINA-2020-full-report_final-_wms.pdf

2. Wheaton AG, Pleasants RA, Croft JB, et al. Gender and asthmachronic obstructive pulmonary disease overlap syndrome. J Asthma 2016;53:720-31. https://doi.org/10.3109/02770903.2016.1154072

3. Patel O, Syamlal G, Wood J, Dodd KE, Mazurek JM. Asthma mortality among persons aged 15-64 years, by industry and occupation-United States, 1999-2016. MMWR Morb Mortal Wkly Rep 2018;67:60-5. https://doi.org/10.15585/mmwr.mm6702a2

4. Ford ES. Trends in mortality from COPD among adults in the United States. Chest 2015;148:962-70. https://doi.org/10.1378/chest.14-2311

5. Lange P, Çolak Y, Ingebrigtsen TS, Vestbo J, Marott JL. Long-term prognosis of asthma, chronic obstructive pulmonary disease, and asthma-chronic obstructive pulmonary disease overlap in the Copenhagen City Heart Study: a prospective population-based analysis. Lancet Respir Med 2016;4:454-62. https://doi.org/10.1016/S2213-2600(16)00098-9

\footnotetext{
$\dagger_{\dagger \dagger}$ https://www.cdc.gov/nchs/data/misc/hb_occup.pdf.
} 
6. Blanc PD, Annesi-Maesano I, Balmes JR, et al. The occupational burden of nonmalignant respiratory diseases. An official American Thoracic Society and European Respiratory Society statement. Am J Respir Crit Care Med 2019;199:1312-34. https://doi.org/10.1164/ rccm.201904-0717ST

7. Baur X, Bakehe P, Vellguth H. Bronchial asthma and COPD due to irritants in the workplace - an evidence-based approach. J Occup Med Toxicol 2012;7:19-50. https://doi.org/10.1186/1745-6673-7-19
8. Calvert GM, Luckhaupt SE, Sussell A, Dahlhamer JM, Ward BW. The prevalence of selected potentially hazardous workplace exposures in the US: findings from the 2010 National Health Interview Survey. Am J Ind Med 2013;56:635-46. https://doi.org/10.1002/ajim.22089

9. White GE, Mazurek JM, Moorman JE. Work-related asthma and employment status - 38 states and District of Columbia, 2006-2009. J Asthma 2013;50:954-9. https://doi.org/10.3109/02770903.2013.829491

10. Camilli AE, Robbins DR, Lebowitz MD. Death certificate reporting of confirmed airways obstructive disease. Am J Epidemiol 1991;133:795-800. https://doi.org/10.1093/oxfordjournals.aje.a115958 